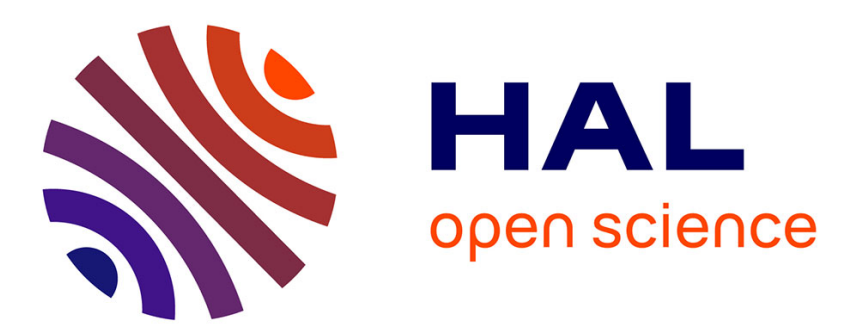

\title{
Array Geometry Impact on Music in the Presence of Spatially Distributed Sources
}

Wenmeng Xiong, José Picheral, Sylvie Marcos

\section{To cite this version:}

Wenmeng Xiong, José Picheral, Sylvie Marcos. Array Geometry Impact on Music in the Presence of Spatially Distributed Sources. Digital Signal Processing, 2017, 63, pp.155-163. 10.1016/j.dsp.2017.01.001 . hal-01349526v2

\section{HAL Id: hal-01349526 \\ https://hal-centralesupelec.archives-ouvertes.fr/hal-01349526v2}

Submitted on 30 Mar 2017

HAL is a multi-disciplinary open access archive for the deposit and dissemination of scientific research documents, whether they are published or not. The documents may come from teaching and research institutions in France or abroad, or from public or private research centers.
L'archive ouverte pluridisciplinaire HAL, est destinée au dépôt et à la diffusion de documents scientifiques de niveau recherche, publiés ou non, émanant des établissements d'enseignement et de recherche français ou étrangers, des laboratoires publics ou privés. 


\title{
ARRAY GEOMETRY IMPACT ON MUSIC IN THE PRESENCE OF SPATIALLY DISTRIBUTED SOURCES
}

\author{
XIONG Wenmeng, PICHERAL José, MARCOS Sylvie \\ Laboratoire des signaux et systèmes, CentraleSupélec-CNRS-Université Paris-Sud, \\ 91192 Gif-sur-Yvette,France
}

\begin{abstract}
The MUltiple SIgnal Classification (MUSIC) estimator has been widely studied for a long time for its high resolution capability in the domain of the direction of arrival (DOA) estimation, with the sources assumed to be point. However, when the actual sources are spatially distributed with angular dispersion, the performance of the conventional MUSIC is degraded. In this paper, the impact of the array geometry on the DOA estimation of spatially distributed sources impinging on a sensor array is considered. Taking into account a coherently distributed source model, we establish closed-form expressions of the MUSIC-based DOA estimation error as a function of the positions of the array sensors in the presence of model errors due to the angular dispersion of the signal sources. The impact of the array geometry is studied and particular array designs are proposed to make DOA estimation more robust to source dispersion. The analytical results are validated by numerical simulations. ${ }^{1}$
\end{abstract}

Keywords: array signal processing, distributed sources, angular dispersion, array geometry, performance, MUSIC

\section{Introduction}

The DOA estimation based on snapshots received on a sensor array has been widely studied with plenty of methods [2]. Among these methods, the MUltiple SIgnal Classification (MUSIC) [3] is famous for its high resolution in the case of point sources. However, in many applications, such as acoustic source imaging [4] and mobile channel communication [5], where angular dispersion of the sources up to $10^{\circ}$ may occur, the physical sources can no longer be considered as points. In this case the performance of the DOA estimation obtained by the conventional MUSIC are degraded, and a spatially distributed model of the sources would be more appropriate.

The models for spatially distributed sources have been classified into two types, namely incoherently distributed (ID) sources and coherently distributed (CD) sources [6]. On one hand, for ID sources, signals coming from different points of the same distributed source can be considered uncorrelated. On the other hand, in the scenario of CD sources, the received signal components are delayed and scaled replicas from different points of the same one [6]. For CD sources, the performances of MUSIC with discretely distributed sources and continuously distributed sources have been investigated in [7], and [8], respectively. As expected, due to the angular dispersion

\footnotetext{
${ }^{1}$ This work has been partially published in [1].
} 
the mismatch between the steering vector model of MUSIC and the actual steering vectors of the sources causes estimation errors. Plenty methods such as the joint estimation of both the DOA and the angular dispersion parameter [6][9][10] have been proposed to solve these problems. However, these methods require a high computational burden or a knowledge of the shape of the source angular distribution for the model, which motivates us to rather keep the conventional point-source MUSIC for the DOA estimation and seek other ways to improve the performances as, for example, the optimization of the array geometry.

The array geometry effect on the DOA estimation has been studied in plenty of publications and in different contexts. The uniform linear array (ULA) is the simplest. However, a more complex geometry can lead to better performance. Optimal array geometries have been designed to reach isotropic and/or optimal performance based on the Cramér-Rao bound (CRB) criterion (eg : $[11,12,13])$, the lower bound of the mean square error (MSE) can be uniform for all the possible DOAs, or the inferior bound of the MSE of the DOA in the elevational and horizontal direction can be decoupled. More recently, based on the spatial aliasing phenomenon, a class of non-uniform array geometries composed of two or more uniform linear arrays (ULAs) with different inter-element spacing has been used to reduce the computational burden of the Maximum Likelihood (ML) estimator [14]. Also, many techniques have been applied in the sparsity array design or large-scale broadband array to reduce the number of elements in an array, to offer a lower cost, power consumption, and heat dissipation (eg : [15] [16] [17]).

In our work, we focus on the impact of the array geometry on the performance of the MUSIC estimator with the CD source model, in the presence of errors due to modeling mismatch of source dispersion. Let us first briefly review our work in [8] and [1]. In [8], the point source MUSIC has been extended into CD-MUSIC (coherent source MUSIC), the first order Talyor approximation DOA estimation bias is proposed in the case that the steering vectors in the model and of the actual sources are mismatched. In [1] we make use of the theoretical estimation bias expression in [8], and express the DOA estimation bias as a function of the sensor positions. Impacts of particular array geometries on the estimator performances are studied in the scenario of single source and multiple sources, respectively. In this paper, besides the results in the conference papers, our contributions consist of: firstly, a general condition for canceling the DOA estimation bias in the case of one source is derived; secondly, the condition for canceling the cross terms of the DOA and the angular dispersion parameter in Cramer-Rao bound in the case of one source is derived; thirdly, for the results concerning UCA in the case of two source in [1], the calculation details are given in 6.1 .

The organization of this paper is as follows. The signal model and a brief recall of MUSIC are given in section 2. The impact of array geometry on the performance of MUSIC and on the crossed terms of the CRB are studied in section 4. Particular geometry designs are studied in section 5. UCA for the case of two sources are studied in section 6. Finally, conclusions are given in section 7 .

\section{Signal model}

Let us consider $q$ spatially CD far-field narrow-band sources impinging on an array of $M$ sensors. The sources arrive from the DOA $\theta_{1}, \ldots, \theta_{q}$, and the position of the $m-t h$ sensor is given by the polar coordinates $\rho_{m}$ and $\alpha_{m}$. Without loss of generality, the signals and the sensors are assumed to be in the same plane, as shown in Figure 1. The $q$ source signals and the $M$ signals received by the array at moment $t$ are denoted by $\mathbf{s}(t)=\left[s_{1}(t), \ldots, s_{q}(t)\right]^{T}$ and $\mathbf{y}(t)=\left[y_{1}(t), \ldots, y_{M}(t)\right]^{T}$, 
respectively. In the case of CD sources, it is common to exploit the model proposed in [6] in order to express the received signal:

$$
\mathbf{y}(t)=\mathbf{C}(\theta) \mathbf{s}(t)+\mathbf{n}(t)
$$

where $\mathbf{n}(t) \in \mathbb{C}^{M \times 1}$ represents the complex Gaussian distributed additive noise, $\mathbf{C}(\theta)=\left[\mathbf{c}_{h_{1}}\left(\theta_{1}\right), \ldots\right.$, $\left.\mathbf{c}_{h_{q}}\left(\theta_{q}\right)\right] \in \mathbb{C}^{M \times q}$ is the array steering matrix composed of $q$ steering vectors $\mathbf{c}_{h_{i}}(\theta)$ that can be written by:

$$
\mathbf{c}_{h_{i}}\left(\theta_{i}\right)=\int_{-\frac{\pi}{2}}^{\frac{\pi}{2}} \mathbf{a}\left(\theta_{i}+\phi\right) h_{i}(\phi) d \phi
$$

where $i=1 \ldots q$, and $\mathbf{a}(\theta)$ is the steering vector for a point source, which can be given by:

$$
\mathbf{a}\left(\theta_{i}\right)=\left[e^{-j 2 \pi \frac{\rho_{1}}{\lambda} \cos \left(\theta_{i}-\alpha_{1}\right)}, \ldots, e^{-j 2 \pi \frac{\rho_{M}}{\lambda} \cos \left(\theta_{i}-\alpha_{M}\right)}\right]^{T},
$$

where $\lambda$ is the wavelength, and $[\cdot]^{T}$ is the transpose operation.

The functions $h_{i}(\phi)$ are introduced in (2) to describe the angular spread distribution (for instance, Uniform and Gaussian distributions). The source signals and the additive noise are considered to be complex centered Gaussian independent random variables. Assuming that signals and noises are uncorrelated and the sources are uncorrelated with each other, the correlation matrix is given by:

$$
\mathbf{R}=E\left[\mathbf{y} \mathbf{y}^{H}\right]=\mathbf{C R}_{s} \mathbf{C}^{H}+\sigma_{b}^{2} \mathbf{I}
$$

where $E[$.$] is the expectation operator, \mathbf{R}_{s}$ and $\sigma_{b}^{2}$ are the source covariance matrix and the noise variance, respectively.

Under the hypothesis that $q<M$ and $\mathbf{R}_{s}$ and $\mathbf{C}$ are not rank deficient, it is well known that the decomposition of $\mathbf{R}$ into eigenvalues $\lambda_{m}$ and eigenvectors $\mathbf{e}_{m}$ is as follows :

$$
\mathbf{R}=\sum_{m=1}^{M} \lambda_{m} \mathbf{e}_{m} \mathbf{e}_{m}=\mathbf{U} \Lambda_{s} \mathbf{U}^{H}+\sigma_{b}^{2} \mathbf{V} \mathbf{V}^{H},
$$

where $\mathbf{U}=\left[\mathbf{e}_{1}, \ldots, \mathbf{e}_{q}\right]$ spans the signal subspace defined by the columns of $\mathbf{C}, \mathbf{V}=\left[\mathbf{e}_{q+1}, \ldots, \mathbf{e}_{M}\right]$ spans the noise subspace defined as the orthogonal complement of $\mathbf{U}$, and $\Lambda_{s}=\operatorname{diag}\left\{\lambda_{1}, \ldots, \lambda_{q}\right\}$.

\section{MUSIC estimator and performances}

The MUSIC [3] method makes use of the orthogonal property of the subspaces spanned by $\mathbf{C}(\theta)$ and $\mathbf{V}$ to estimate the DOAs $\theta_{i}$. In practice it is difficult to know exactly the angular dispersion of the actual sources, consequently, the steering vector model of the point source $\mathbf{a}(\theta)$ is used here instead of $\mathbf{c}_{h_{i}}(\theta)$ to estimate the value of $\theta$ :

$$
\hat{\theta}_{i}=\underset{\theta}{\operatorname{argmax}} \frac{1}{\left\|\mathbf{a}^{H}(\theta) \mathbf{V}\right\|^{2}} .
$$

Here, we assume that the number of snapshots is large enough such that the estimation error of the noise subspace can be neglected and the DOA estimation error comes mainly from the model error, it is to say, the mismatch of the angular dispersion parameter between the model of the MUSIC estimator $\mathbf{a}(\theta)$ and the actual source $\mathbf{c}_{h_{i}}\left(\theta_{i}\right)$. We recall here the standard analysis in 
order to express the estimation error $\Delta \theta_{i}=\hat{\theta}_{i}-\theta_{i}, \hat{\theta}_{i}$ should satisfy that the first derivative of denominator in (6) is null:

$$
\left.\frac{\partial \mathbf{a}(\theta)^{H} \mathbf{V} \mathbf{V}^{H} \mathbf{a}(\theta)}{\partial \theta}\right|_{\hat{\theta}_{i}}=0
$$

which gives:

$$
2 \mathcal{R} e\left\{\dot{\mathbf{a}}\left(\hat{\theta}_{i}\right)^{H} \mathbf{V} \mathbf{V}^{H} \mathbf{a}\left(\hat{\theta}_{i}\right)\right\}=0
$$

where $\dot{\mathbf{a}}\left(\hat{\theta}_{i}\right)=\left.\frac{\partial \mathbf{a}(\theta)}{\partial \theta}\right|_{\hat{\theta}_{i}}$.

Assuming that, $\hat{\theta}_{i}$ is not far away from $\theta_{i}$, we make the first order approximation of Taylor:

$$
\mathbf{a}\left(\hat{\theta}_{i}\right) \approx \mathbf{a}\left(\theta_{i}\right)+\Delta \theta_{i} \dot{\mathbf{a}}\left(\theta_{i}\right),
$$

and:

$$
\dot{\mathbf{a}}\left(\hat{\theta}_{i}\right) \approx \dot{\mathbf{a}}\left(\theta_{i}\right)+\Delta \theta_{i} \ddot{\mathbf{a}}\left(\theta_{i}\right)
$$

where $\ddot{\mathbf{a}}\left(\theta_{i}\right)=\left.\frac{\partial \dot{\mathbf{a}}(\theta)}{\partial \theta}\right|_{\theta_{i}}$.

Note $\Delta \mathbf{c}\left(\theta_{i}\right)=\mathbf{a}\left(\theta_{i}\right)-\mathbf{c}\left(\theta_{i}\right)$ the error on the steering vector due to the mismatch between the estimator model and the actual signals. Note also $\Delta \mathbf{V}=\tilde{\mathbf{V}}-\mathbf{V}$ the error on the noise eigenvectors matrix, where $\tilde{\mathbf{V}}$ is the noise eigenvectors matrix which would appear if the steering vectors $\mathbf{a}\left(\theta_{i}\right)$ would replace $\mathbf{c}\left(\theta_{i}\right)$ in (1), where $i=1, \ldots, q$. Introducing (9) and (10) into (8), and keeping only the first order of $\Delta \theta_{i}$, the DOA estimation error for source $\theta_{i}$ can be expressed as:

$$
\Delta \theta_{i}=\frac{\mathcal{R} e\left\{\dot{\mathbf{a}}_{i}^{H} \tilde{\Pi} \mathbf{c}_{i}\right\}}{\dot{\mathbf{a}}_{i}^{H} \tilde{\Pi} \dot{\mathbf{a}}_{i}},
$$

where $\tilde{\Pi}=\mathbf{I}-\tilde{\mathbf{V}} \tilde{\mathbf{V}}^{H}$ is the noise subspace projector of the steering vector model of the estimator, which would correspond to the case of point sources, $\mathbf{I}$ is the $M \times M$ identity matrix. Note that $\theta_{i}$ is omitted in $\dot{\mathbf{a}}\left(\theta_{i}\right), \mathbf{a}\left(\theta_{i}\right)$ and $\mathbf{c}\left(\theta_{i}\right)$ which are now noted $\dot{\mathbf{a}}_{i}, \mathbf{a}_{i}$ and $\mathbf{c}_{i}$, respectively.

\section{Array geometry analyses for one source}

In this section, we firstly investigate the array geometry condition for MUSIC to be robust to the source angular dispersion. Then, we link and compare our results with the existing results based on the CRB for an isotropic array in the case of point source. At last, we derive the CRB for joint estimation of the DOA and the angular distribution in the scenario of a distributed source.

\subsection{Array geometry for MUSIC}

In this subsection, we study the estimation error of the point source MUSIC estimator defined in (6) in the case where there is only one source arriving from the DOA $\theta$. Notice that in this case $\tilde{\Pi}=\mathbf{I}-\frac{1}{M} \mathbf{a}_{1} \mathbf{a}_{1}^{H}$. From (11) the DOA estimation error can then be given by:

$$
\Delta \theta=\frac{\mathcal{R} e\left\{\dot{\mathbf{a}}^{H} \mathbf{c}-\frac{1}{M} \dot{\mathbf{a}}^{H} \mathbf{a} \mathbf{a}^{H} \mathbf{c}\right\}}{\dot{\mathbf{a}}^{H} \dot{\mathbf{a}}-\frac{1}{M}\left|\dot{\mathbf{a}}^{H} \mathbf{a}\right|^{2}},
$$

where $\mathbf{c}_{1}$ and $\mathbf{a}_{1}$ have been replaced by $\mathbf{c}$ and $\mathbf{a}$. In the following, we wish to express the estimation error as an explicit function of the sensor positions, so as to study the impact of the sensor array geometry on the performance of MUSIC. 


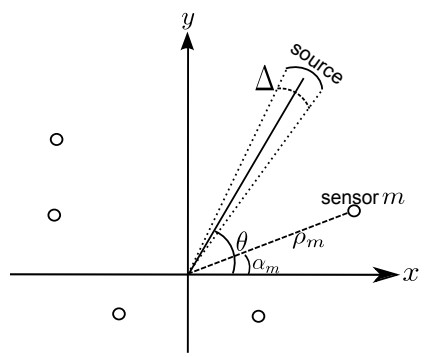

Figure 1: Planar array and source DOAs

For the $m-t h$ sensor as illustrated in Figure 1, let us define:

$$
\begin{aligned}
& \varphi_{m}=2 \pi \frac{\rho_{m}}{\lambda} \cos \left(\theta-\alpha_{m}\right), \\
& \dot{\varphi}_{m}=-2 \pi \frac{\rho_{m}}{\lambda} \sin \left(\theta-\alpha_{m}\right), \\
& \ddot{\varphi}_{m}=-2 \pi \frac{\rho_{m}}{\lambda} \cos \left(\theta-\alpha_{m}\right), \\
& u_{m}=\int \cos \left(\varphi_{m}(\theta+\phi)-\varphi_{m}(\theta)\right) h(\phi) d \phi, \\
& v_{m}=\int \sin \left(\varphi_{m}(\theta+\phi)-\varphi_{m}(\theta)\right) h(\phi) d \phi .
\end{aligned}
$$

Assuming that the angular dispersion of the distributed source is small enough with a symmetrical distribution, we introduce a third order Taylor approximation in $\phi$ of $\varphi_{m}(\theta+\phi)-\varphi_{m}(\theta)$ so as the last equation in (13) yields:

$$
v_{m} \approx \frac{1}{2} \ddot{\varphi}_{m} \sigma^{2}
$$

where:

$$
\sigma^{2} \triangleq \int \phi^{2} h(\phi) d \phi
$$

remark that $\sigma$ is the angular dispersion of the source signal.

Introducing the notations (13) in (12), $\Delta \theta$ can be given by:

$$
\Delta \theta=\frac{\overline{\dot{\varphi} \cdot v}-\bar{\varphi} \cdot \bar{v}}{\overline{\dot{\varphi}^{2}}-(\overline{\dot{\varphi}})^{2}}
$$

where $\overline{\text { ? }}$ is operator to calculate the "average" of a deterministic series such that $\bar{x}=\frac{1}{M} \sum_{m=1}^{M} x_{m}$. Eq (16) makes it possible to design the geometry in order to minimize $\Delta \theta$.

Let us introduce (14) in (16), $\Delta \theta$ can be given by:

$$
\Delta \theta=\frac{1}{2} \sigma^{2} \frac{\overline{\dot{\varphi} \cdot \ddot{\varphi}}-\bar{\varphi} \cdot \ddot{\varphi}}{\bar{\varphi}^{2}-(\bar{\varphi})^{2}}
$$

We can observe that this expression of the DOA estimation error is proportional to the square of the source angular dispersion $\sigma^{2}$. 
From (17), the estimation error as a function of $x_{m}$ and $y_{m}$ can be derived as:

$$
\Delta \theta=\frac{1}{4} \sigma^{2} \cdot \frac{\sin (2 \theta)\left[\overline{x^{2}}-(\bar{x})^{2}-\overline{y^{2}}+(\bar{y})^{2}\right]-\cos (2 \theta)(\overline{x y}-\bar{x} \bar{y})}{\sin ^{2} \theta\left[\overline{x^{2}}-(\bar{x})^{2}\right]+\cos ^{2} \theta\left[\overline{y^{2}}-(\bar{y})^{2}\right]-\sin (2 \theta)[\overline{x y}-\bar{x} \bar{y}]} .
$$

The sufficient and necessary condition for $\Delta \theta$ to be zero for any value of $\theta$ is given by:

$$
\left\{\begin{array}{l}
\overline{x^{2}}-(\bar{x})^{2}=\overline{y^{2}}-(\bar{y})^{2} \\
\overline{x y}=\bar{x} \bar{y}
\end{array}\right.
$$

If the condition (19) is verified then the modelling mismatch introduced in (6) by using point source steering vector does not introduce an estimation error. Despite the fact that the sensor coordinates are deterministic, one can give a statistical interpretation, $\overline{x y}=\bar{x} \bar{y}$ means that the sensors' the abscissa and ordinates are uncorrelated, and $\overline{x^{2}}-(\bar{x})^{2}=\overline{y^{2}}-(\bar{y})^{2}$ means that the standard deviation on abscissa and ordinate are the same. Geometrically, this means that the sensor position should not exhibit a specific direction and that the spread of the sensor position is the same in all the directions.

Notice that (19) can be exploited to improve an existing array by adding (or removing) one sensor such that the condition (19) is verified.

Figure 2 illustrates an example of the optimal geometry of the sensor array obtained by minimizing numerically the criterion $\int_{0}^{2 \pi}|\Delta \theta| d \theta$, where $\Delta \theta$ is given in (18), in this example we have a source with a uniform angular distribution $10^{\circ}$. The numerical "greedy research" method proposed in [12] is used here to avoid the ambiguity problem. In this case, condition (19) is verified, since: $\overline{x^{2}}-(\bar{x})^{2}=\overline{y^{2}}-(\bar{y})^{2} \approx 0.49, \overline{x y}=\bar{x} \bar{y} \approx 0.21$.

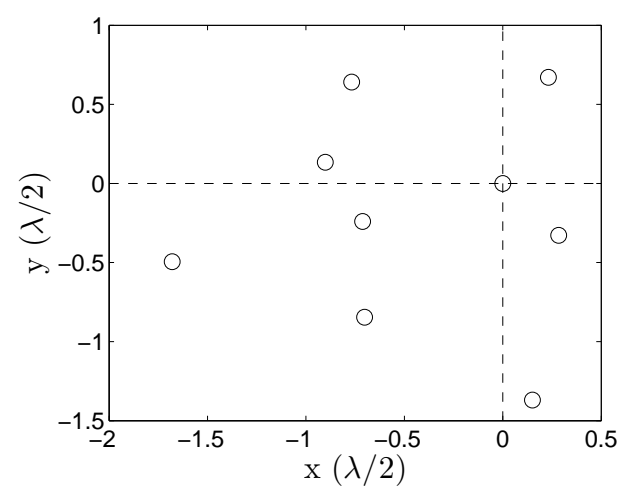

Figure 2: one example of a nine-element optimal array for MUSIC

\subsection{Link with the Cramer-Rao bound}

Firstly, one can note that the condition to obtain an isotropic array in the scenario of point source proposed in [11] is identical to (19). Note that (19) has been established in the presence of a model error due to the sources dispersion and an infinite number of snapshots to estimate the covariance matrix whereas the expression in [11] corresponds to the point sources case and the error is due to a finite number of snapshots. 
Secondly, we investigate the CRB for the joint estimator of the DOA and the source angular dispersion. We now consider, the scenario with one distributed source, where the shape of angular distribution $h_{i}$ is known but the angular dispersion parameter $\sigma$ is unknown and has to be jointly estimated with the DOA. An example of such an estimator is the $2 D$ MUSIC-like estimator named DSPE [6]. Under the unconditional-model assumption (UMA) [18], the CRB matrix is composed of the derivatives of the elements in the parameter vector $\Phi_{0}=\left[\theta, \sigma, \sigma_{s}^{2}, \sigma_{b}^{2}\right]$. We will compute the CRB in this case, and express the condition such that the lower bound on the covariance of the estimation of $\theta$ and $\sigma$ is null. When this condition is fullfilled, the consistent estimator is known to be robust to the model error due to the source dispersion since $\operatorname{cov}(\tilde{\theta}, \tilde{\sigma}) \underset{N \rightarrow \infty}{\rightarrow} 0$, where $\tilde{\theta}=\hat{\theta}-\theta, \tilde{\sigma}=\hat{\sigma}-\sigma$. In other words, error on $\sigma$ does not impact on the $\hat{\theta}$ estimation.

It is well known that for point sources, the variances of parameters of signal and noise power are decoupled of the variance of the DOA. Similar results can be found for distributed sources when the angular dispersion is small [19]. Therefore, without loss of generality, we assume that $\sigma_{s}^{2}$ and $\sigma_{b}^{2}$ are known. The CRB expression for distributed sources has been derived in [19]:

$$
C R B\left(\theta, \sigma \mid \sigma_{s}^{2}, \sigma_{b}^{2}\right)=\frac{1+\beta S N R}{2 N \beta S N R}\left[\mathcal{R} e\left\{\mathbf{C}_{\Psi}^{H} \Pi \mathbf{C}_{\Psi}\right\}+\Omega\right]^{-1},
$$

where $\beta=\mathbf{c}^{H} \mathbf{c}, \Omega=\frac{2 \mathbf{b b}^{T}}{\beta(1+\beta S N R)}, \mathbf{b}=\mathcal{R} e\left\{\mathbf{C}_{\Psi}^{H} \mathbf{c}\right\}, \mathbf{C}_{\Psi}=\left[\frac{\partial \mathbf{c}}{\partial \theta}, \frac{\partial \mathbf{c}}{\partial \sigma}\right]$ and $S N R$ is the value of the signal to noise ratio.

Exploiting the approximation proposed in (14), we develop the CRB expression as an explicit function of the parameters:

$$
\begin{aligned}
C R B_{\theta \theta} & =\frac{f_{\sigma \sigma}}{\eta\left[f_{\theta \theta} f_{\sigma \sigma}-\left|f_{\theta \sigma}\right|^{2}\right]}, \\
C R B_{\sigma \sigma} & =\frac{4}{\sigma_{\sigma}^{2} \eta} \cdot \frac{f_{\theta \theta}}{f_{\theta \theta} f_{\sigma \sigma}-\left|f_{\theta \sigma}\right|^{2}}, \\
C R B_{\theta \sigma} & =\frac{2}{\sigma_{\sigma} \eta} \cdot \frac{f_{\theta \sigma}}{f_{\theta \theta} f_{\sigma \sigma}-\left|f_{\theta \sigma}\right|^{2}},
\end{aligned}
$$

where:

$$
\begin{aligned}
\sigma_{\sigma} & \triangleq \int \phi^{2} \frac{\partial h(\phi)}{\partial \sigma} d \phi=\frac{\partial \sigma^{2}}{\partial \sigma}=2 \sigma, \\
\eta & =\frac{2 N \sigma_{s}^{4}}{\sigma_{b}^{2}\left(\sigma_{s}^{2}\left(M+\sigma^{2} \mathcal{R e a l}\left\{\ddot{\mathbf{a}}^{H} \mathbf{a}\right\}\right)+\sigma_{b}^{2}\right)}, \\
f_{\theta \theta} & =M \dot{\mathbf{a}}^{H} \dot{\mathbf{a}}-\left|\dot{\mathbf{a}}^{H} \mathbf{a}\right|^{2}+\sigma^{2} \mathcal{R} e a l\left\{\ddot{\mathbf{a}}^{H} \dot{\mathbf{a}} M+\dot{\mathbf{a}}^{H} \ddot{\mathbf{a}}^{H} \mathbf{a}-\mathbf{a}^{H} \dot{\mathbf{a}}\left(\dddot{\mathbf{a}}^{H} \mathbf{a}+\dot{\mathbf{a}}^{H} \ddot{\mathbf{a}}\right)\right\}, \\
f_{\theta \sigma} & =\mathcal{R e a l}\left\{M \dot{\mathbf{a}}^{H} \ddot{\mathbf{a}}-\dot{\mathbf{a}}^{H} \mathbf{a a}^{H} \ddot{\mathbf{a}}\right\}, \\
f_{\sigma \sigma} & =M \ddot{\mathbf{a}}^{H} \ddot{\mathbf{a}}+\mathcal{R} \operatorname{Real}\left\{\mathbf{a}^{H} \ddot{\mathbf{a}}^{H} \ddot{\mathbf{a}}\right\}-\frac{2 M \sigma_{s}^{2} \mathcal{R e a l}\left\{\ddot{\mathbf{a}}^{H} \mathbf{a}\right\}^{2}}{\sigma_{b}^{2}+\sigma_{s}^{2}\left(M+\sigma^{2} \mathcal{R e a l}\left\{\ddot{\mathbf{a}}^{H} \mathbf{a}\right\}\right)} .
\end{aligned}
$$

Taking into account the notations in (13), $f_{\theta \sigma}$ can be rewritten as:

$$
f_{\theta \sigma}=M^{2}(\overline{\dot{\varphi} \cdot \ddot{\varphi}}-\overline{\dot{\varphi}} \cdot \bar{\varphi}) .
$$

As discussed previously, in order to design array geometry for which consistent DOA estimators are robust to mismodelling, the condition is $f_{\theta \sigma}=0$. From (25) it yields $\overline{\dot{\varphi} \cdot \ddot{\varphi}}=\overline{\dot{\varphi}} \cdot \bar{\varphi}$, we can 
see that the results of (19), (25), and the results in [18] are identical. Notice that (19) yields the condition for MUSIC to be robust to the source spatial dispersion whereas (25) corresponds to a CRB criterion for a model of source dispersion and that the condition in [11] has been derived through the CRB calculus for point sources. It follows that the optimal design criterion obtained for MUSIC in the case of spatially distributed sources yields to the same solution as those obtained by $\mathrm{CRB}$ both in the distributed and point cases.

\section{Particular array geometries design}

In this section, we investigate particular array geometries with pretty simple structures which satisfy the optimal condition proposed in (19) in the case of one source.

\subsection{Uniform circular array}

Inspired by (17), we find that for a UCA with an even number of symmetrical elements, where $\rho_{m}=\rho$, and $\alpha_{m}=2 \pi \frac{(m-1)}{M}$ with $M$ even, $\overline{\dot{\varphi}}$ and $\overline{\dot{\varphi} \cdot \ddot{\varphi}}$ can be zero:

$$
\begin{aligned}
\overline{\dot{\varphi}} & =-\frac{1}{M} 2 \pi \frac{\rho}{\lambda} \sum_{m=1}^{M} \sin \left(\theta-\alpha_{m}\right) \\
& =-\frac{1}{M} 2 \pi \frac{\rho}{\lambda}\left[\sum_{m=1}^{\frac{M}{2}} \sin \left(\theta-\alpha_{m}\right)+\sum_{m=1}^{\frac{M}{2}} \sin \left(\theta-\alpha_{m}+\pi\right)\right] \\
& =0
\end{aligned}
$$

and similarly :

$$
\overline{\dot{\varphi} \cdot \ddot{\varphi}}=\frac{\left(2 \pi \frac{\rho}{\lambda}\right)^{2}}{M} \sum_{m=1}^{M} \underbrace{\sin \left(\theta-\alpha_{m}\right) \cos \left(\theta-\alpha_{m}\right)}_{\frac{1}{2} \sin \left(2 \theta-2 \alpha_{m}\right)}=0 .
$$

This result illustrates that a symmetrical with even elements UCA can be robust to the model error due to the source dispersion, in the scenario of one source. In addition, a sensor array composed of a combination of UCAs with such a structure with a same center but different radii, can also be proved, with a similar demonstration, to be robust to the model error.

\subsection{V-shape array}

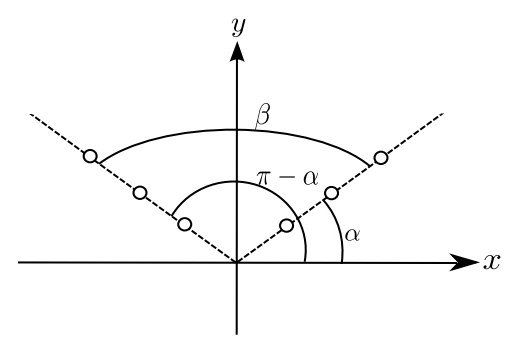

Figure 3: Illustration of V-shape arrays

The V-shape array (VA) is another type of particular geometry which has been widely studied, thanks to its easiness to be parameterized. It has been proved in [12] that with the array angle 
fixed to a specific value, a VA with any size can have an isotropic behavior, and outperforms the $\mathrm{UCA}$ in terms of CRB.

Let us assume that, without loss of generality, the two identical branches are symmetrical with respect to the $y$ axis, with no sensor placed at the origin, as depicted in Figure 3, and that, the inter-sensor distance is fixed to $d$. The coordinates of the sensors can be expressed as:

$$
\rho_{m}=\left\{\begin{array}{ll}
m d, & 1 \leqslant m \leqslant \frac{M}{2} \\
\left(m-\frac{M}{2}\right) d, & \frac{M}{2} \leqslant m \leqslant M
\end{array},\right.
$$

and,

$$
\alpha_{m}=\left\{\begin{array}{lr}
\alpha, & 1 \leqslant m \leqslant \frac{M}{2} \\
\pi-\alpha, & \frac{M}{2} \leqslant m \leqslant M
\end{array} .\right.
$$

Considering the above described V-shape geometry, (17) can be derived as:

$$
\Delta \theta=\frac{1}{2} \sigma^{2} \frac{\sin \theta \cos \theta\left[(4+4 M) \cos ^{2} \alpha+(2-M) \sin ^{2} \alpha\right]}{(4+4 M) \cos ^{2} \alpha \sin ^{2} \theta+(2-M) \sin ^{2} \alpha \cos ^{2} \theta}
$$

which shows that the DOA estimation error is independent of $d$. In addition, we can see that $\Delta \theta$ can be zero, under the condition that the numerator of (30) vanishes, which yields:

$$
\tan ^{2} \alpha=\frac{4 M+4}{M-2} .
$$

This expression reveals that the array angular $\alpha$ can be fixed to a special value to be robust to the model error due to the source dispersion, which depends roughly on the array size, and is robust to other parameters. For example, in the case $M=10$ with 5 sensors on each branch, (31) yields $\alpha \approx 66.9^{\circ}$ and $\beta \approx 46.2^{\circ}$.

\subsection{Linear array}

Notice that the scenario of $\alpha_{m}=0$ corresponds to the linear array (LA). After some straightforward calculus from (13)-(17) with $\alpha_{m}=0$, the DOA estimation error is derived as:

$$
\Delta \theta=\frac{1}{2} \sigma^{2} \cot \theta
$$

which reveals that in the case of LA, the DOA estimation error does not depend on the sensor positions but only on the DOA and the square of the angular dispersion of the source $\sigma^{2}$. Taking into account that the model error can not be modified, the sensor array should be rotated to reduce the value of $\cot \theta$, so as to get a smaller estimation error, if $\theta$ is roughly known. Besides, this result is identical to the conclusion that we have for point sources: it is better to have the DOA perpendicular to the array than in the axis of the array.

\subsection{Simulation results}

In this subsection, we validate the theoretical results through numerical experiments in the scenario of one source. In all experiments, one uniformly distributed source with a support within $\left[\theta-\frac{\Delta_{0}}{2}, \theta+\frac{\Delta_{0}}{2}\right]$ is considered, where $\Delta_{0}=10^{\circ}$ is the angular width of the source, for uniform distribution $\sigma^{2}=\frac{\Delta_{0}^{2}}{12}$. Signals received on the sensor array are simulated with $N=1000$ snapshots 
and 100 Monte-Carlo experiments, with $S N R=10 \mathrm{~dB}$. The UCA are composed of $M=20$ sensors. The VA are composed of 10 sensors identically uniformly placed at the two branches, spaced by $d=\lambda / 2$. In this case, the optimal VA corresponds to $\alpha=65.2^{\circ}$ or equivalently $\beta=49.7^{\circ}$. The VA with $\alpha=0$ reduces to a LA. Such array configurations ensure that the UCA and the LA have the same horizontal aperture.

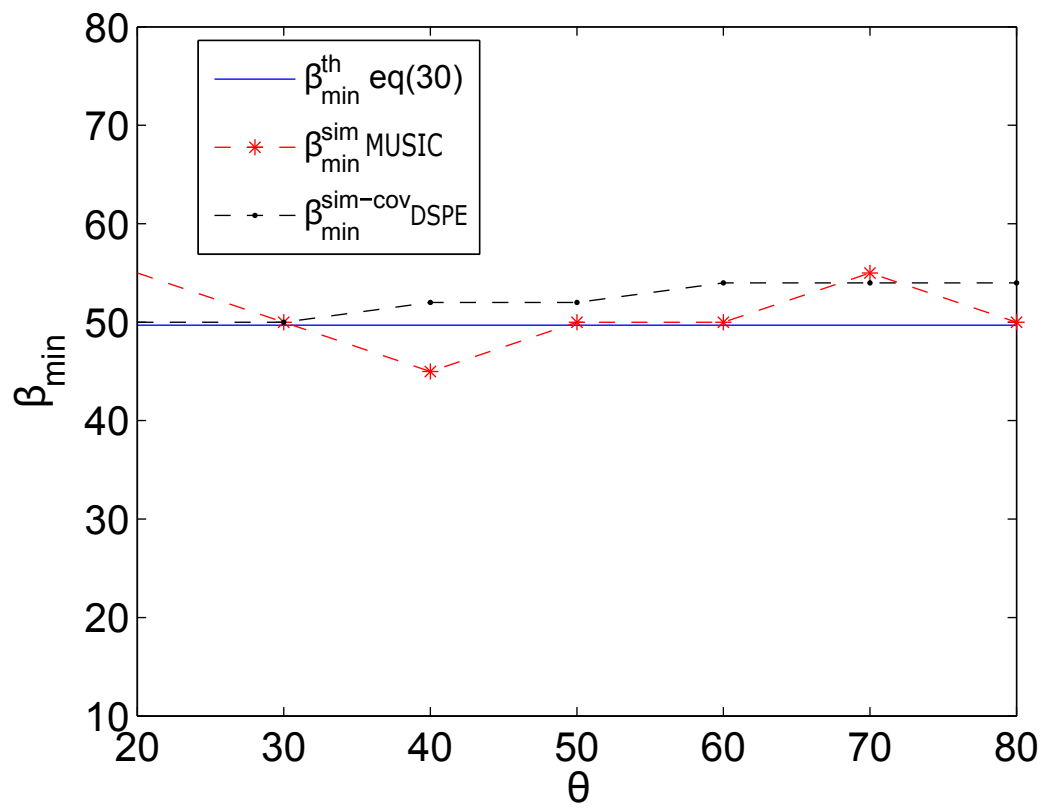

Figure 4: Value of $\beta$ corresponds to the minimum value of: 1)the theoretical expression of $|\Delta \theta|$ in eq(30), 2)the $|\Delta \theta|$ obtained by the simulation with MUSIC 3)the covariance of $\theta$ and $\sigma$ of DSPE (simulation), (VA with $M=10$ and $d=\lambda / 2$, one source with uniform angular dispersion, $\left.\Delta_{0}=10^{\circ}\right)$

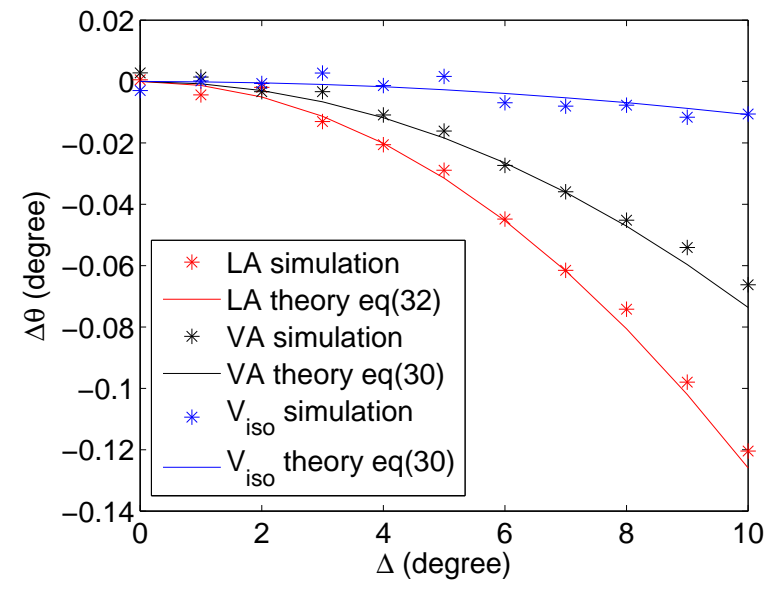

Figure 5: DOA estimation error vs. the source angular extension $\Delta_{0}$ (LA with $M=20$ and $d=\lambda / 4$, VA with $M=20$ and $d=\lambda / 4$ and $\beta=100^{\circ}$, $\mathrm{V}_{\text {iso }}$ with $M=20$ and $d=\lambda / 4$ and $\beta=53.13^{\circ}$, one source with uniform angular dispersion with $\left.\theta=45^{\circ}, \Delta_{0}=2 \sqrt{3} \sigma\right)$ 
In Figure 4, we consider a V-shaped array and compare the theoretical value of the optimal $\beta$ to the value obtained by simulation: $\beta_{\text {min }}^{\text {sim }}$ MUSIC is the value of $\beta$ which minimizes the estimation error of MUSIC for 100 trials. This result can be similarly obtained by exploiting the cross term of the CRB as proposed in section 4.1. To illustrate this, we also plot the value of $\beta$ corresponding to the minimum $|\operatorname{Cov}(\theta \sigma)|=|E[(\hat{\theta}-\theta)(\hat{\sigma}-\sigma)]|$ obtained by the joint estimator DSPE [6]. Let us recall the definition of the DSPE, assuming that the distribution shape of the source is known, the DOA and the angular dispersion parameter can be estimated as:

$$
\{\hat{\theta}, \hat{\sigma}\}=\underset{\theta, \sigma}{\operatorname{argmax}} \frac{1}{\left\|\mathbf{c}_{h}^{H}(\theta) \mathbf{V}\right\|^{2}} .
$$

As one may expect, for a $\mathrm{V}$-shape array, the optimal geometry for an $1 D$ estimator is robust to the DOA of the source, in addition, this optimal geometry makes it possible to decouple the estimation of the DOA and the angular dispersion parameter for a $2 D$ estimator.

In Figure 5, the DOA estimation error of MUSIC versus the angular dispersion of the signal source with $\theta=45^{\circ}$ is presented, in the case of LA, VA with $\beta=100^{\circ}$ and VA with $\beta=53.13^{\circ}$ $\left(\mathrm{V}_{\text {iso }}\right)$. The $\mathrm{V}$-shape array $\mathrm{V}_{\text {iso }}$ proposed in [13] satisfies the isotropic condition for the CRB in the scenario of a point source and also corresponds to the solution of (31) when M tends to infinity or at least becomes large. The $\Delta \theta$ with array $\mathrm{V}_{\text {iso }}$ is the smallest among the three arrays, because $\beta=53.13^{\circ}$ is close to $\beta_{\text {min }}^{\text {th }}$ in Figure 4 . We can observe the validation of expression (30) and (32) which makes it possible to consider the DOA estimation error as an explicit convex quadratic function of the angular dispersion of the signal source. Furthermore, regardless of a more complicated geometry, the two VAs outperforms LA, which reveals the interest to optimize the array geometry to have a better performance.

\section{Two sources in the case of a UCA}

\subsection{Theoretical results}

In this subsection, we focus on the above-mentioned case of a UCA with an even number of symmetrically positioned sensors, which is easier to manipulate mathematically comparing to other geometries.

Assuming two sources arriving from $\theta_{1}$ and $\theta_{2}$, with $\theta_{2}>\theta_{1}$. For the $m-t h$ sensor, let us introduce the notation:

$$
\Delta \varphi_{m}=\varphi_{m}\left(\theta_{2}\right)-\varphi_{m}\left(\theta_{1}\right)=\varphi_{m, 2}-\varphi_{m, 1} .
$$

Notice that with a UCA:

$$
\mathcal{R} e\left\{\dot{\mathbf{a}}_{i}^{H} \mathbf{a}_{i}\right\}=-\sum_{m=1}^{M} 2 \pi \frac{\rho}{\lambda} \sin \left(\theta-\alpha_{m}\right)=0,
$$

and:

$$
\mathcal{R} e\left\{\dot{\mathbf{a}}_{i}^{H} \mathbf{c}_{i}\right\}=\sum_{m=1}^{M} \dot{\varphi}_{m, i} u_{m, i}=\left[\sum_{m=1}^{M / 2} \Phi\left(\theta_{i}\right)+\sum_{m=1}^{M / 2} \Phi\left(\theta_{i}+\pi\right)\right]=0,
$$

where $\Phi\left(\theta_{i}\right)=\int \dot{\varphi}_{m}\left(\theta_{i}\right)\left[\cos \left(\varphi_{m}\left(\theta_{i}+\phi\right)\right) \cos \left(\varphi_{m}\left(\theta_{i}\right)\right)+\sin \left(\varphi_{m}\left(\theta_{i}+\phi\right)\right) \sin \left(\varphi_{m}\left(\theta_{i}\right)\right)\right] h(\phi) d \phi$. 
Introducing (35), (36), and the steering matrix $\mathbf{A}=\left[\mathbf{a}_{1}, \mathbf{a}_{2}\right]$ in (11) gives:

$$
\Delta \theta_{1}=\frac{\mathcal{R} e\left\{-M \dot{\mathbf{a}}_{1}^{H} \mathbf{a}_{2} \mathbf{a}_{2}^{H} \mathbf{c}_{1}+\dot{\mathbf{a}}_{1}^{H} \mathbf{a}_{2} \mathbf{a}_{1}^{H} \mathbf{c}_{1} \mathbf{a}_{2}^{H} \mathbf{a}_{1}\right\}}{\dot{\mathbf{a}}_{1}^{H} \dot{\mathbf{a}}_{1}\left(M^{2}-\left|\mathbf{a}_{2}^{H} \mathbf{a}_{1}\right|^{2}\right)-M\left|\dot{\mathbf{a}}_{1}^{H} \mathbf{a}_{2}\right|^{2}} .
$$

We introduce the following notations: $\overline{u_{(i)}}=\frac{1}{M} \sum_{m=1}^{M} u_{m, i}, \overline{v_{(i)}}=\frac{1}{M} \sum_{m=1}^{M} v_{m, i}, \overline{\varphi_{(i)}}=$ $\frac{1}{M} \sum_{m=i}^{M} \varphi_{m, i}$, where $u_{m, i}, v_{m, i}$, and $\varphi_{m, i}$ are simplified for $u_{m}\left(\theta_{i}\right), v_{m}\left(\theta_{i}\right), \varphi_{m}\left(\theta_{i}\right)$, respectively. $i=1,2$ in the case of two sources. Introducing the notations of (13) and (34) in (37), with the results in appendix $\mathrm{A}, \Delta \theta_{1}$ can be derived as:

$$
\Delta \theta_{1}=-\frac{1}{2} \sigma^{2} \frac{\left(18 \overline{\dot{\varphi}_{(1)}^{2}}-3 \delta^{2} \overline{\dot{\varphi}_{(1)}^{2}+\dot{\varphi}_{(1)}^{4}}\right) \overline{\left(\dot{\varphi}_{(1)}^{2}-\overline{\dot{\varphi}_{(1)}^{2}}\right)^{2}}}{\delta\left[-9\left(\overline{\dot{\varphi}_{(1)}^{2}}\right)^{3}+12 \overline{\dot{\varphi}_{(1)}^{2}} \cdot \overline{\dot{\varphi}_{(1)}^{4}+\dot{\varphi}_{(1)}^{2}}-\delta^{2}\left(\overline{\dot{\varphi}_{(1)}^{2}+\dot{\varphi}_{(1)}^{4}}\right)^{2}\right]},
$$

where $\delta=\theta_{2}-\theta_{1}$. We can see that (38) is proportional to the square of the angular dispersion $\sigma^{2}$. The value of the estimation error is inversely proportional to the value of $\delta$ for small $\delta$, which illustrates that the estimation error decreases as the sources move away from each other.

In order to explore the peak separation, let us consider the sign of $\Delta \theta_{1}$. Firstly, $\tilde{\Pi}$ is Hermitian, so the denominator $\dot{\mathbf{a}}_{i}^{H} \tilde{\Pi}_{i} \dot{\mathbf{a}}_{i}$ in (11) is non-negative, it is enough to focus on the numerator in (11) for $\theta_{1}$ which yields $\frac{1}{2} \sigma^{2} \delta^{3}\left(18 \overline{\dot{\varphi}_{(1)}^{2}}-3 \delta^{2} \overline{\dot{\varphi}_{(1)}^{2}+\dot{\varphi}_{(1)}^{4}} \overline{\left(\dot{\varphi}_{(1)}^{2}-\overline{\dot{\varphi}_{(1)}^{2}}\right)^{2}}\right.$ (see (A.5) in appendix) after some straightforward calculus. Secondly with a small $\delta, 18 \overline{\dot{\varphi}_{(1)}^{2}}-3 \delta^{2} \overline{\dot{\varphi}_{(1)}^{2}+\dot{\varphi}_{(1)}^{4}}$ can be approximated by $18 \overline{\dot{\varphi}_{(1)}^{2}}$ which is positive, and obviously $\overline{\left(\dot{\varphi}_{(1)}^{2}-\overline{\dot{\varphi}_{(1)}^{2}}\right)^{2}}$ is positive. Therefore, in the case that $\theta_{1}<\theta_{2}, \Delta \theta_{1}$ is opposite to the sign of $\delta$.

For $\theta_{2}$, after similar calculus in appendix $\mathrm{B}$, we have:

$$
\Delta \theta_{2}=-\Delta \theta_{1}
$$

$\Delta \theta_{1}$ is negative and $\Delta \theta_{2}$ is positive, on the opposite of the well known result for point source, the lower DOA is underestimated and the other one is overestimates, in consequence, the two peaks move away. This implies that when the two sources are close to each other, MUSIC is always able to give the estimation of two DOAs separately, despite that the value of the estimation error increases as it is illustrated in Figure 6.

\subsection{Numerical results}

In this section, we validate the theoretical results through numerical experiments in the scenario of two sources. In all experiments, two uniformly sources with the angular dispersion $\Delta_{0}=10^{\circ}$ are considered. Signals received on the sensor array are simulated with $N=1000$ snapshots and 100 Monte-Carlo experiments, with $S N R=10 \mathrm{~dB}$. The array configurations are the same for the UCA, VA and LA as in subsection 5.4.

Figure 6 illustrates the peak separation of criterion MUSIC in the case of 2 sources. A UCA is used for the DOA estimation of two uniformly distributed sources coming from $29.5^{\circ}$ and $30.5^{\circ}$ with angular dispersion $10^{\circ}$, assuming that the correlation matrix is perfectly known. Two peaks can be detected, but for arguments which are different from the actual DOAs. The analyses of the signs of the two sources in section 6.1 can be verified in this figure.

In Figure 7, we compare the performance of MUSIC with the UCA and with the LA versus the DOA separation, in the scenario of two sources. The DOA separation varies with $\theta_{c}=\frac{1}{2}\left(\theta_{1}+\theta_{2}\right)$ 


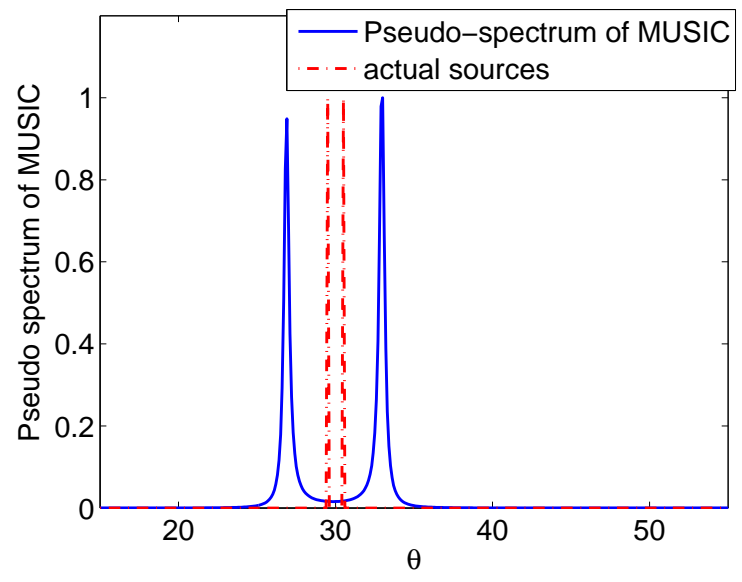

Figure 6: Pseudo-spectrum of MUSIC for two distributed sources with a UCA

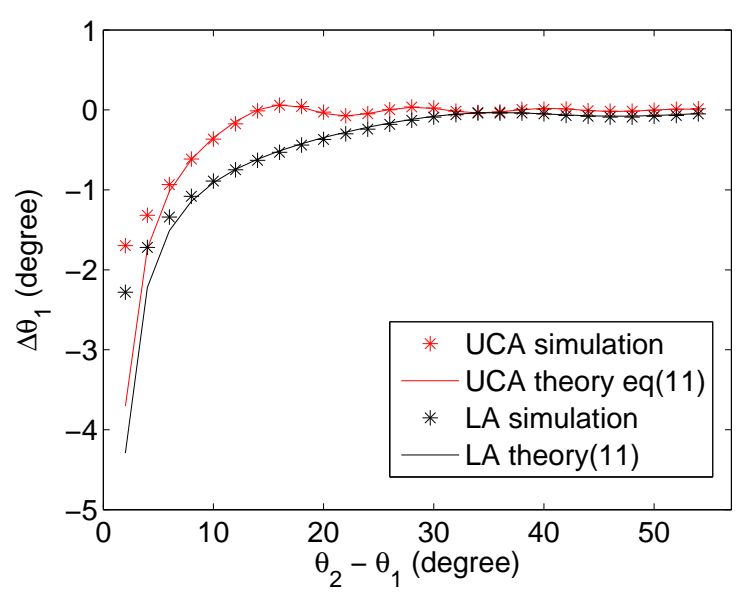

(a) $\theta_{1}$

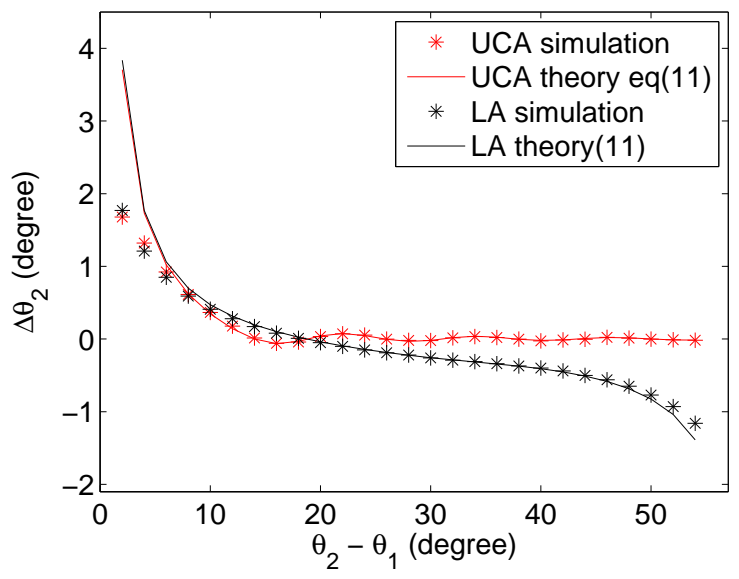

(b) $\theta_{2}$

Figure 7: DOA estimation error vs. DOA separation (UCA with $M=20$, LA with $M=20$ and $d=\lambda / 4$, two sources with uniform angular dispersion, $\left.\Delta_{0}=10^{\circ}, \theta_{c}=\frac{1}{2}\left(\theta_{1}+\theta_{2}\right)=60^{\circ}\right)$ 


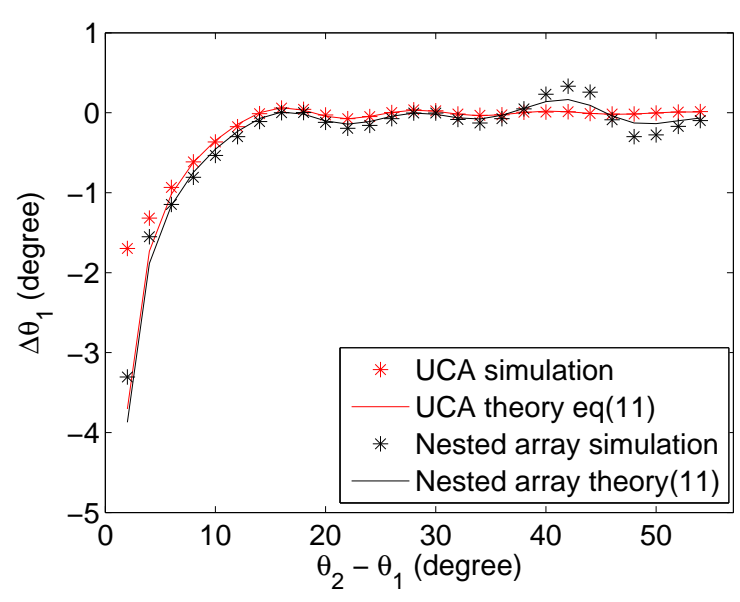

(a) $\theta_{1}$

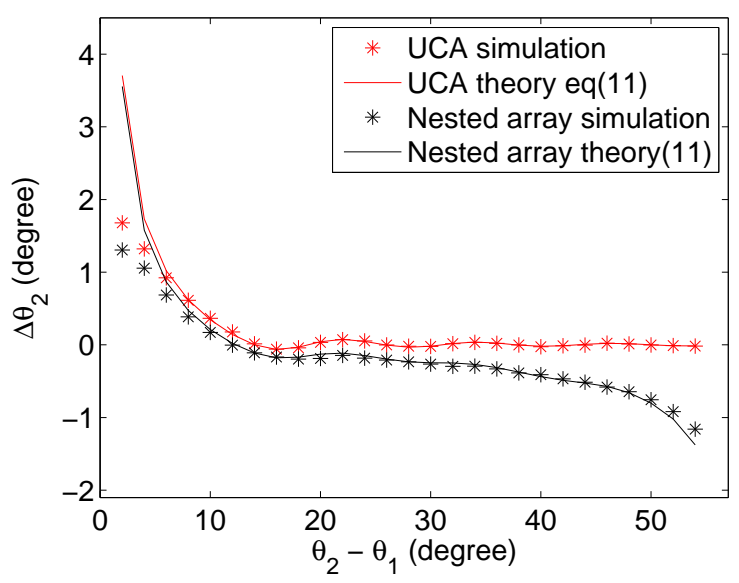

(b) $\theta_{2}$

Figure 8: DOA estimation error vs. DOA separation (UCA with $M=20$, nested array with $M=8$ and $d_{1}=\lambda / 2$, $d_{2}=3 \lambda / 2$, two sources with uniform angular dispersion, $\left.\Delta_{0}=10^{\circ}, \theta_{c}=\frac{1}{2}\left(\theta_{1}+\theta_{2}\right)=60^{\circ}\right)$

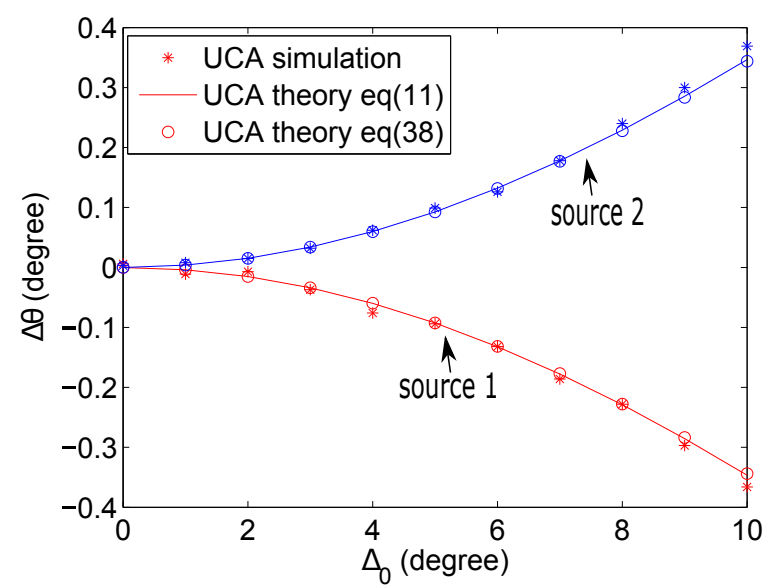

Figure 9: DOA estimation error vs. the source angular extension $\Delta_{0}$ (UCA with $M=20$, two sources with uniform angular dispersion, $\theta_{1}=55^{\circ}, \theta_{2}=65^{\circ}$ ) 
fixed to $60^{\circ}$, assuming that $\theta_{2}>\theta_{1}$. We can see that when the two sources are too close to each other, the estimation error can not be ignored despite the array geometry, due to the interference between the sources. For LA, $\Delta \theta_{1}$ decreases as the DOA separation increases and as $\theta_{1}$ tends to zero, by contrast, $\Delta \theta_{2}$ decreases as the interference phenomenon fades, but increases again along with the DOA separation, which coincides with the well-known conclusion that the ULA has a better performance for broadside sources. For UCA, both $\Delta \theta_{1}$ and $\Delta \theta_{2}$ tend to zero quickly as the DOA separation increases, which is in adequacy with the scenario of one source where the DOA estimation error is null and outperforms its LA counterpart. In addition, it is interesting to see the validity of the approximated expression (38) and (39) for describing the trend and the sign of the estimation error when the sources are close to each other.

In Figure 8, we compare the performance of MUSIC with the UCA and a two-level nested array proposed in [20] [21]. The nested array is composed of $M=8$ sensors, the four sensors in the first level are spaced by $d_{1}=\lambda / 2$, and the last four sensors in the second level are spaced by $d_{1}=3 \lambda / 2$. Therefore, the nested array has a same array aperture as the UCA. The advantage of UCA with respect to the nested array is mainly illustrated when the angular separation between the two sources becomes larger, in this case, MUSIC with UCA has a better performance than nested array, especially for $\theta_{2}$.

In Figure $9, \Delta \theta_{1}$ and $\Delta \theta_{2}$ is plotted versus the angular extension of the sources, two sources of $\theta_{1}=55^{\circ}$ and $\theta_{2}=65^{\circ}$ are used in this experiment. The goal is to validate the expression (38) as an explicit function of the angular extension $\Delta_{0}$ (recall that for uniform source $\sigma^{2}=\frac{\Delta_{0}^{2}}{12}$ ).

\section{Conclusion}

In this paper, we have studied the impact of the array geometry on the performance of the MUSIC DOA estimation in the presence of spatially distributed sources. We have found that the DOA estimation error can be reduced and even canceled for particular array geometries in the case of one source or in the case where the DOA separation between two sources is large enough. Additionally, the impact of the array geometry on the crossed terms of CRB has been studied, and the condition for decoupling the estimation of the DOA from the angular dispersion parameter has been found the same as the one for canceling the DOA estimation error of MUSIC. The separation property of MUSIC in the scenario of two distributed sources has been explored with a UCA, and motivates the further investigation of the array geometry design in the scenario of two sources.

\section{Appendix A.}

Introducing (13) and (34) in (37), $\Delta \theta_{1}$ is given as:

$$
\Delta \theta_{1}=\frac{\mathcal{R} e\left\{\overline{j \dot{\varphi}_{(1)} e^{-j \Delta \varphi}} \cdot \overline{e^{j \Delta \varphi}\left(u_{(1)}-j v_{(1)}\right)}-\overline{j \dot{\varphi}_{(1)} e^{-j \Delta \varphi}} \cdot \overline{\left(u_{(1)}-j v_{(1)}\right)} \cdot \overline{e^{j \Delta \varphi}}\right\}}{\overline{\dot{\varphi}_{(1)}^{2}}\left(1-\left\|\overline{e^{-j \Delta \varphi}}\right\|^{2}\right)-\left\|\overline{\dot{\varphi}_{(1)} e^{-j \Delta \varphi}}\right\|^{2}} .
$$

where $\overline{e^{-j \Delta \varphi}}=\frac{1}{M} \sum_{m=1}^{M} e^{-j \Delta \varphi_{m}}, \overline{e^{j \Delta \varphi}\left(u_{(1)}-j v_{(1)}\right)}=\frac{1}{M} \sum_{m=1}^{M} e^{j \Delta \varphi_{m}}\left(u_{m, 1}-j v_{m, 1}\right)$. 
Noting that $e^{-j \Delta \varphi_{m}}=\cos \left(\Delta \varphi_{m}\right)-j \sin \left(\Delta \varphi_{m}\right)$, we have:

$$
\begin{aligned}
\overline{\sin (\Delta \varphi)} & =\frac{1}{M} \sum_{m=1}^{M} \sin \left(\Delta \varphi_{m}\right)=\frac{1}{M} \sum_{1}^{M}\left[\sin \left(2 \pi \frac{\rho}{\lambda} \cos \left(\theta_{2}-\alpha_{m}\right)\right) \cos \left(2 \pi \frac{\rho}{\lambda} \cos \left(\theta_{1}-\alpha_{m}\right)\right)\right. \\
& \left.-\cos \left(2 \pi \frac{\rho}{\lambda} \cos \left(\theta_{2}-\alpha_{m}\right)\right) \sin \left(2 \pi \frac{\rho}{\lambda} \cos \left(\theta_{1}-\alpha_{m}\right)\right)\right] \\
= & \frac{1}{M}\left\{\sum _ { 1 } ^ { \frac { M } { 2 } } \left[\sin \left(2 \pi \frac{\rho}{\lambda} \cos \left(\theta_{2}-\alpha_{m}\right)\right) \cos \left(2 \pi \frac{\rho}{\lambda} \cos \left(\theta_{1}-\alpha_{m}\right)\right)\right.\right. \\
& \left.-\cos \left(2 \pi \frac{\rho}{\lambda} \cos \left(\theta_{2}-\alpha_{m}\right)\right) \sin \left(2 \pi \frac{\rho}{\lambda} \cos \left(\theta_{1}-\alpha_{m}\right)\right)\right]- \\
& \sum_{1}^{\frac{M}{2}}\left[\sin \left(2 \pi \frac{\rho}{\lambda} \cos \left(\theta_{2}-\alpha_{m}+\pi\right)\right) \cos \left(2 \pi \frac{\rho}{\lambda} \cos \left(\theta_{1}-\alpha_{m}+\pi\right)\right)\right. \\
& \left.\left.-\cos \left(2 \pi \frac{\rho}{\lambda} \cos \left(\theta_{2}-\alpha_{m}+\pi\right)\right) \sin \left(2 \pi \frac{\rho}{\lambda} \cos \left(\theta_{1}-\alpha_{m}+\pi\right)\right)\right]\right\}=0
\end{aligned}
$$

Similarly, $\overline{v_{(i)}}$ and $\overline{\dot{\varphi}_{(i)} \cdot \cos (\Delta \varphi)}$ equal to 0 . Considering the terms which equal to $0,(\mathrm{~A} .1)$ yields:

$$
\Delta \theta_{1}=\frac{\overline{\dot{\varphi}_{(1)} \cdot \sin (\Delta \varphi)}\left[\overline{\cos (\Delta \varphi)} \cdot \overline{u_{(1)}}-\overline{\cos (\Delta \varphi) \cdot u_{(1)}}+\overline{\sin (\Delta \varphi) \cdot v_{(1)}}\right]}{\overline{\dot{\varphi}_{(1)}^{2}}-\overline{\dot{\varphi}_{(1)}^{2}} \cdot \overline{\cos ^{2}(\Delta \varphi)}-\left(\overline{\dot{\varphi}_{(1)} \sin (\Delta \varphi)}\right)^{2}} .
$$

We take the third order of Taylor approximation in $\delta$ to develop (B.1) rather than the first order, because the angular separation between the sources can be up to $50^{\circ}-60^{\circ}$. Hence, noting that $\delta=\theta_{2}-\theta_{1}$, the approximated $\sin \left(\Delta \varphi_{m}\right)$ and $\cos \left(\Delta \varphi_{m}\right)$ can be given by:

$$
\begin{aligned}
\sin \left(\Delta \varphi_{m}\right) & \approx \Delta \varphi_{m}-\frac{1}{6} \Delta \varphi_{m}^{3} \\
& =2 \pi \frac{\rho}{\lambda}\left[\cos \left(\theta_{1}+\alpha_{m}\right)\left(\delta-\frac{1}{6} \delta^{3}\right)-\frac{1}{2} \sin \left(\theta_{1}+\alpha_{m}\right) \delta^{2}\right]-\frac{1}{6}\left(2 \pi \frac{\rho}{\lambda}\right)^{3} \cos ^{3}\left(\theta_{1}+\alpha_{m}\right) \delta^{3} \\
\cos \left(\Delta \varphi_{m}\right) & \approx 1-\frac{1}{2} \Delta \varphi_{m}^{2} \\
& =1-\frac{1}{2}\left(2 \pi \frac{\rho}{\lambda}\right)^{2}\left[\cos ^{2}\left(\theta_{1}+\alpha_{m}\right) \delta^{2}-\cos \left(\theta_{1}+\alpha_{m}\right) \sin \left(\theta_{1}+\alpha_{m}\right) \delta^{3}\right] .
\end{aligned}
$$

Introducing (A.4) in (B.1), $\Delta \theta_{1}$ results in:

$$
\begin{aligned}
& \Delta \theta_{1}= \frac{\delta^{3}\left(18 \overline{\dot{\varphi}_{(1)}^{2}}-3 \delta^{2} \overline{\dot{\varphi}_{(1)}^{2}+\dot{\varphi}_{(1)}^{4}}\right)\left(\overline{\dot{\varphi}_{(1)}^{2} \cdot u_{(1)}}-\overline{\dot{\varphi}_{(1)}^{2}} \cdot \overline{u_{(1)}}\right)}{\delta^{4}\left[-9\left(\overline{\dot{\varphi}_{(1)}^{2}}\right)^{3}+12 \overline{\dot{\varphi}_{(1)}^{2}} \cdot \overline{\dot{\varphi}_{(1)}^{4}+\dot{\varphi}_{(1)}^{2}}-\delta^{2}\left(\overline{\dot{\varphi}_{(1)}^{2}+\dot{\varphi}_{(1)}^{4}}\right)^{2}\right]} \\
&=\frac{\left(18 \overline{\dot{\varphi}_{(1)}^{2}}-3 \delta^{2} \overline{\dot{\varphi}_{(1)}^{2}+\dot{\varphi}_{(1)}^{4}}\right)\left(\overline{\dot{\varphi}_{(1)}^{2} \cdot u_{(1)}}-\overline{\dot{\varphi}_{(1)}^{2}} \cdot \overline{u_{(1)}}\right)}{\delta\left[-9\left(\overline{\dot{\varphi}_{(1)}^{2}}\right)^{3}+12 \overline{\bar{\varphi}_{(1)}^{2}} \cdot \overline{\dot{\varphi}_{(1)}^{4}+\dot{\varphi}_{(1)}^{2}}-\delta^{2}\left(\overline{\dot{\varphi}_{(1)}^{2}+\dot{\varphi}_{(1)}^{4}}\right)^{2}\right]} .
\end{aligned}
$$

Taking into consideration that $u_{m, 1} \approx \int \cos \left(\varphi_{m}\left(\theta_{1}+\phi\right)-\varphi_{m}\left(\theta_{1}\right)\right) h(\phi) d \phi \approx \int \cos \left(\phi \dot{\varphi}_{m}\left(\theta_{1}\right)+\right.$ $\left.\frac{1}{2} \phi^{2} \ddot{\varphi}_{m}\left(\theta_{1}\right)+\frac{1}{6} \phi^{2} \dddot{\varphi}_{m}\left(\theta_{1}\right)\right) h(\phi) d \phi \approx 1-\frac{1}{2} \sigma^{2} \dot{\varphi}_{m}^{2}\left(\theta_{1}\right), \Delta \theta_{1}$ results in (38) 


\section{Appendix B.}

Similarly to (A.1), $\Delta \theta_{2}$ can be given as:

$$
\Delta \theta_{2}=\frac{\mathcal{R} e\left\{\overline{j \dot{\varphi}_{(2)} e^{j \Delta \varphi}} \cdot \overline{e^{-j \Delta \varphi}\left(u_{(2)}-j v_{(2)}\right)}-\overline{j \dot{\varphi}_{(2)} e^{j \Delta \varphi}} \cdot \overline{\left(u_{(2)}-j v_{(2)}\right)} \cdot \overline{e^{-j \Delta \varphi}}\right\}}{\overline{\dot{\varphi}_{(2)}^{2}}\left(1-\left\|\overline{e^{-j \Delta \varphi}}\right\|^{2}\right)-\|{\overline{\dot{\varphi}}(2) e^{-j \Delta \varphi}}_{\|^{2}}^{2}} .
$$

Recalling that $\theta_{2}=\theta_{1}+\delta$, the notations in $\theta_{2}$ can be approximated as functions of notations in $\theta_{1}$ and $\delta$ :

$$
\begin{gathered}
\dot{\varphi}_{m, 2}=-2 \pi \frac{\rho_{m}}{\lambda} \sin \left(\theta_{1}+\delta-\alpha_{m}\right) \\
\approx \dot{\varphi}_{m, 1}-2 \pi \frac{\rho_{m}}{\lambda}\left[-\frac{1}{2} \delta^{2} \sin \left(\theta_{1}-\alpha_{m}\right)+\left(\delta-\frac{1}{6} \delta^{3}\right) \cos \left(\theta_{1}-\alpha_{m}\right)\right] \\
v_{m, 2} \approx \frac{1}{2} \ddot{\varphi}_{m, 2} \sigma^{2} \\
\approx v_{m, 1}-\pi \sigma^{2} \frac{\rho_{m}}{\lambda}\left[-\frac{1}{2} \delta^{2} \cos \left(\theta_{1}-\alpha_{m}\right)-\left(\delta-\frac{1}{6} \delta^{3}\right) \sin \left(\theta_{1}-\alpha_{m}\right)\right] \\
u_{m, 2}=\int \cos \left[\varphi_{m}\left(\theta_{1}+\phi+\delta\right)-\varphi_{m}\left(\theta_{1}+\delta\right)\right] h(\phi) d \phi \\
\approx u_{m, 1}-\quad(\operatorname{B} .2) \\
\int\left(\cos \left[2 \pi \frac{\rho_{m}}{\lambda}\left(\cos \left(\theta_{1}+\phi\right)-\cos \theta_{1}\right)\right]\left[\left(\pi \frac{\rho_{m}}{\lambda}\right)^{2}\left(\delta^{2} \phi \sin \theta_{1}+\delta \phi^{2} \sin \theta_{1}-2 \delta \phi \cos \theta_{1}\right)\right]\right. \\
\left.+\sin \left(2 \pi \frac{\rho_{m}}{\lambda}\left(\cos \left(\theta_{1}+\phi\right)-\cos \theta_{1}\right)\right) \sin \left(2 \pi \frac{\rho_{m}}{\lambda}\left(-\delta^{2} \phi \sin \theta_{1}-\delta \phi^{2} \sin \theta_{1}+2 \delta \phi \cos \theta_{1}\right)\right)\right) h(\phi) d \phi
\end{gathered}
$$

Putting (B.2), (B.3), and (B.4) in (B.1), and keeping the third order of Taylor approximation in $\delta$, the final expression of $\Delta \theta_{2}$ results in (39).

[1] Wenmeng Xiong, Jose Picheral, and Sylvie Marcos. Antenna array design for music in the presence of spatially distributed sources. In Signal Processing Conference (EUSIPCO), 2015 23rd European, pages 1371-1375. IEEE, 2015 .

[2] H. Krim and M. Viberg. Two decades of array signal processing research: the parametric approach. Signal Processing Magazine, IEEE, 13(4):67-94, 1996.

[3] P. Stoica and N. Arye. Music, maximum likelihood, and cramer-rao bound. Acoustics, Speech and Signal Processing, IEEE Trans. on, 37(5):720-741, 1989.

[4] N. Chu, A.. Djafari, and J. Picheral. Robust Bayesian super-resolution approach via sparsity enforcing a priori for near-field aeroacoustic source imaging. Journal of Sound and Vibration, 332(18):4369-4389, 2013.

[5] K.I.Pedersen, P.E.Mogensen, and B.H.Fleury. A stochastic model of the temporal and azimuthal dispersion seen at the base station in outdoor propagation environments. IEEE Transaction on Vehicular Technology, 49(2), Mar. 2000.

[6] S.Valaee, B.Champagne, and P.Kabal. Parametric localization of distributed sources. IEEE Trans. on Signal Processing, 43(9):2144-2153, 1995.

[7] D. Astély and B. Ottersten. The effects of local scattering on direction of arrival estimation with music. Signal Processing, IEEE Trans. on, 47(12):3220-3234, 1999.

[8] Wenmeng Xiong, Jose Picheral, and Sylvie Marcos. Performance analysis of music in the presence of modeling errors due to the spatial distributions of sources. In Acoustics, Speech and Signal Processing (ICASSP), 2015 IEEE International Conference on, pages 2804-2808. IEEE, 2015. 
[9] J. Abou Chaaya, J. Picheral, and S. Marcos. Localization of spatially distributed near-field sources with unknown angular spread shape. Signal Processing, 106:259-265, 2015.

[10] A.Zoubir, Y.Wang, and Pascal Chargé. Efficient subspace-based estimator for localization of multiple incoherently distributed sources. IEEE Transaction on Signal Processing, 56(2):532-542, Feb. 2008.

[11] U. Baysal and R. Moses. On the geometry of isotropic arrays. Signal Processing, IEEE Trans. on, 51(6):1469$1478,2003$.

[12] H. Gazzah and K. Abed-Meraim. Optimum ambiguity-free directional and omnidirectional planar antenna arrays for doa estimation. Signal Processing, IEEE Trans. on, 57(10):3942-3953, 2009.

[13] H. Gazzah and S. Marcos. Cramer-rao bounds for antenna array design. Signal Processing, IEEE Trans. on, 54(1):336-345, 2006.

[14] J. Shin, Y. Lee, and H. Kim. Reduced-complexity maximum likelihood direction-of-arrival estimation based on spatial aliasing. Signal Processing, IEEE Trans. on, 62(24):6568-6581, 2014.

[15] G. Oliveri and A. Massa. Bayesian compressive sampling for pattern synthesis with maximally sparse nonuniform linear arrays. Antennas and Propagation, IEEE Trans. on, 59(2):467-481, 2011.

[16] Ahmed El-Makadema, Layali Rashid, and Anthony K Brown. Geometry design optimization of large-scale broadband antenna array systems. Antennas and Propagation, IEEE Transactions on, 62(4):1673-1680, 2014.

[17] Carine El Kassis, José Picheral, and Chafic Mokbel. Advantages of nonuniform arrays using root-music. Signal Processing, 90(2):689-695, 2010.

[18] Petre Stoica and Arye Nehorai. Performance study of conditional and unconditional direction-of-arrival estimation. Acoustics, Speech and Signal Processing, IEEE Transactions on, 38(10):1783-1795, Oct. 1990.

[19] Jason Goldberg and Hagit Messer. Inherent limitations in the localization of a coherently scattered source. Signal Processing, IEEE Transactions on, 46(12):3441-3444, Dec. 1998.

[20] Piya Pal and PP Vaidyanathan. Nested arrays: A novel approach to array processing with enhanced degrees of freedom. IEEE Transactions on Signal Processing, 58(8):4167-4181, 2010.

[21] Keyong Han and Arye Nehorai. Nested array processing for distributed sources. IEEE Signal Processing Letters, 21(9):1111-1114, 2014. 